\title{
Analysis of Peripherally Inserted Central Catheter- Related Complications and Causes: a Retrospective Study of 2974 Children Blood Disease Patients in Single Center in China
}

\section{Li-Xian Chang}

Institute of Hematology \& Blood Diseases Hospital

Yu-Wen Chen

Institute of Hematology \& Blood Diseases Hospital

Meng-Chuan Wang

Institute of Hematology \& Blood Diseases Hospital

Su-Yu Zhao

Institute of Hematology \& Blood Diseases Hospital

Min Wang

Institute of Hematology \& Blood Diseases Hospital

Yang Tian

Institute of Hematology \& Blood Diseases Hospital

Li Tang

Institute of Hematology \& Blood Diseases Hospital

Jun-Xia Wang

Institute of Hematology \& Blood Dieseases Hospital

Miao-Miao Yang

Institute of Hematology \& Blood Diseases Hospital

Xiao-Fan Zhu

Institute of Hematology \& Blood Diseases Hospital

Hui-Min Zhang ( $\square$ zhanghuimin@ihcams.ac.cn )

Institute of Hematology \& Blood Diseases Hospital

Research article

Keywords: PICC, risk, rash, catheter, phlebitis

Posted Date: August 18th, 2020

DOI: https://doi.org/10.21203/rs.3.rs-51175/v1 
License: (c) (i) This work is licensed under a Creative Commons Attribution 4.0 International License. Read Full License 


\section{Abstract}

Background: The peripherally inserted central catheter (PICC) has been widely used. However, there is still a lack of large sample size-based relevant risk factor investigation in children with blood diseases in china.

Methods: We performed a retrospective, the single-center cohort study of child blood disease patients with PICC insertion. Totally, 2974 patients were enrolled for our study.

Results: B-ultrasound plus Seldinger technology significantly improved the success rate of PICC insertion. The most common non-infectious complications were rash, followed by catheter blockage, mechanical phlebitis, and catheter broke. the male, 1-3 years old, Power PICC solo catheter and spring are risk factors for the rash. The power PICC solo catheter was the most important risk factor for catheter blockage. Insertion site under the elbow was the most important risk factor for phlebitis and catheter damage.

Conclusion: Our finding first shed new light on the risk factors associated with PICC complications for Chinese blood disease children.

\section{Background}

The peripherally inserted central catheter (PICC) significantly reduces the number of venipunctures in children with chemotherapy or long-term treatment, and the damage to blood vessels caused by chemotherapy drugs, which drastically relieve the pain of children during treatment. Although the PICC has been widely used in the clinic, however, the success rate of related operations and complications, such as phlebitis, rash, and catheter broken after PICC implantation still occur. PICC catheterization has been widely used in clinical practice with a history of more than 80 years. In the late 1990s, PICC was introduced into China and developed rapidly, widely used in tumor chemotherapy, nutritional pathway establishment of premature infants, and tumor chemotherapy for children [1]. It is widely used in children with blood diseases that require long-term chemotherapy and special immunosuppressive agents, but there is still no large number of cases reported in China. So the analysis of the related risk factors of complications is essential. Herein, we, through analyzing different operation methods, the factors affecting the success rate of PICC implantation were summarized. Combined with catheter material, puncture method, insertion site, gender, age, season, and the possible risk factors of different postoperative complications were analyzed.

\section{Methods}

\section{Patients}

A retrospective, the single-center, cohort study of child blood disease patients with PICC insertion was performed to determine the incidence and risk factors associated with PICC complications and the rate of PICC insertion success. 2,974 patients with hematological diseases who underwent PICC placement in 
Chinese academy of medical sciences blood disease hospital children's blood disease diagnosis and treatment center during 2004.3-2018.12. We tried to find the critical factor for the successful PICC operation and the risk factors for postoperative complications; we comprehensively analyzed the success rate of PICC operation, the occurrence and type of complications after PICC placement, the development status of PICC approaches or materials in our center.

\section{PICC operating procedures and principles}

Catheters were inserted using the sterile technique at the bedside or operating room by qualified PICC nurses. Before placing the tube, the puncture arm should be thoroughly cleaned with chlorhexidine disinfectant. PICC catheter types of technology as the technology advances, catheter method including the Non-assistive technique (blind insertion) (550 cases), ultrasound (48 cases) and plug technology (1067), plug dingle + ultrasound-guided puncture technology (1309), we will compare the success rate of catheter methods for clinical provide a higher success rate of catheter method; The puncture position of the catheter is under the elbow and above the elbow. The types of catheters include Groshong ${ } N X T$ ClearVue ${ }^{\mathrm{TM}}$ Catheter 4F (4F), Groshong ${ }^{\circledR}$ Catheter $3 \mathrm{~F}$ (3F), and Power PICC SOLO ${ }^{2 \mathrm{TM}}$ catheter (Power PICC SOLO). Generally, the catheter type is selected according to the principle that the ratio of the catheter to vessel diameter(A/B) is $<45 \%$ (Fig. S1). There are two kinds of catheter materials, silicone material (3F and $4 \mathrm{~F}$ ) and polyurethane material (Power PICC solo). We will provide a data basis for the clinical selection of catheter types and materials by analyzing the differences between different insertion sites, catheter materials, and the incidence of later complications. The length of the external catheter is generally $4-5 \mathrm{~cm}$, of which $2 \mathrm{~cm}$ is in the decompression sleeve. All PICCs were routinely maintained weekly by PICC nurses using sterile technique. The catheters were flushed with $10 \mathrm{~mL}$ normal saline and then sealed with $2 \mathrm{~mL}$ heparin saline (50-100 IU) according to institutional maintenance protocol. In the case of contamination, film crimping, and loosening, the catheter can be nursed at any time.

\section{Complication definition}

Complications were monitored and treated by clinicians and PICC nurses. The common complications in our center included phlebitis, catheter occlusion, accidental withdrawal, breakage, and skin allergy. Rash, Catheter occlusion, breakage, or phlebitis was defined according to Infusion Nursing Standards of Practice [2].

\section{Statistical analysis}

The date was analyzed using the chi-square test and multivariable logistic regression models. These results were presented as adjusted odds ratios (ORs) with $95 \%$ confidence intervals (Cls). All statistical tests were two-sided, and $p$ values $<0.05$ were considered statistically significant. Statistical analysis was performed with SPSS software (IBM SPSS statistics 20).

\section{Results}


During March 2004 to December 2018, 2974 child blood disease patients with PICC in our center. The mean age was 6.2 years (range, 0.1 to 18 years), and $60.4 \%(n=1797)$ of patients were male, $39.6 \%(n=$ $1177)$ patients were female. $76 \%(n=2259)$ had Acute lymphoblastic leukemia (ALL), $11.7 \%(n=349)$ had Acute myeloblastic leukemia(AML), 2.2\% $(n=64)$ had Acute promyelocytic leukemia $(A P L), 0.07 \%(n=2)$ had Acute heterozygotic leukemia (HAL), $1.2 \%(n=36)$ had lymphoma, $0.1 \%(n=4)$ had Myelodysplastic syndrome (MDS), $0.03 \%(n=1)$ had Haemophilic cell syndrome(HPS), $0.06 \%(n=2)$ had Juvenile chronic granular mononuclear leukemia(JMML). 8.5\%(n=255) had Sever aplastic anemia(SAA), $0.03 \%(n=1)$ had Hemolytic anemia (HA). The patients of ALL, APL, AML, HAL, MDS, HPS and JMML underwent chemotherapy, the patients of SAA and HA underwent Immunosuppressive therapy (IST).

\section{B-ultrasound plus Seldinger technology can significantly improve the success rate of PICC insertion}

The success rate of four different operation techniques was analyzed, including no assistive technology (A), simple B-ultrasound (B), simple Seldinger technology (C), and B-ultrasound plus Seldinger technology (D). As shown in Table 1, The success rate of group D (99.7\%) and C (98.9\%) are higher than in other groups, with significant statistical difference compared with group $A(p=0.00)$. The success rate of group $D$ is the highest.

\section{Analysis of risk factors of common complications}

The common complications in our center included rash (453 cases, $15.2 \%$ ), catheter blockage (433 cases, 14.6\%), phlebitis (282 cases, 9.5\%), and PICC catheter broken (164 cases, 5.5\%). Rare complications included total catheter detachment (34 cases, $1.1 \%$ ), catheter rupture (intravascular 2 cases, $0.06 \%$; Extravascular 9 cases, $0.3 \%$ ). The specific values and annual incidence trends are shown in Fig. 1. Here, we mainly analyzed the risk factors of common complications such as rash, catheter blockage, phlebitis, and PICC catheter broken in our center. Phlebitis and catheter broken peak occurred mainly in 2004 to 2013, decreased significantly after 2014 (Fig. 1), considering that the biggest technical change of PICC catheterization in our center since 2014 is that the insertion site is mainly changed from the lower elbow joint to the upper elbow joint, it is preliminarily estimated that may be phlebitis and catheter broken related to the position of catheterization. Fig. 1 shows that the peak of catheter blockage and rash occurred in 2014, and the peak value was in 2015 and 2016, respectively. Considering that the Power PICC solo catheter was used in our center at that time, the possible reason was related to the type of catheter. Therefore, in this paper, we combined the gender, age, puncture location, PICC type, and season to conduct a comprehensive analysis, looking for relevant risk factors of common complications.

1) Analysis of risk factors for rash:

Chi-square test (Table S1) and logistic analysis (Table 2) showed that males were more likely to have rash than females, and the incidence of rash in children aged 1-3 was highest than other groups, and the incidence of rash was significantly different from that in children aged 7-18, with a statistically significant difference. The incidence of Power PICC (30.7\%) was the highest, followed by $3 F(18.4 \%)$ and $4 F(9.6 \%)$. The most rashes occurred in spring (28.5\%), followed by summer(27.8\%), autumn(23\%) and 
winter(20.8\%). The incidence of rashes in spring was statistically different from that in winter. In the chisquare test, the insertion site under the elbow is more likely to have a rash than above the elbow. However, the removal of other factors in logistic analysis suggests that the placement of the catheter is not a risk factor for skin rash. The results suggested that male, 1-3 years old, Power PICC solo catheter and spring were risk factors for the rash occurrence, among which Power PICC solo catheter was the most important risk factor for rash (OR 3.235, 95\% Cl: 2.255-4.642).

2) Risk factors associated with PICC catheter blockage

Catheter blockage occurred in 433 cases. Chi-square analysis (Table S2) showed that age, insertion site, and catheter type were related to catheter occlusion, and the rate of catheter occlusion was the highest in children aged $11-18$ years old(19.6\%), followed by 1-3 years old (15.1\%), 7-10 years old (14\%), and 4-6 years old (12.1\%). Power PICC solo catheters have the highest catheter blockage rate $(47.7 \%)$, with the statistical significance of other PICC types. The results of the Logistic regression analysis (Table 3 ) indicated that the Power PICC solo catheter was the most important risk factor for catheter blockage (OR 4.982, 95\% Cl: 3.817-6.504).

3) Results of risk factors for phlebitis

There were 283 cases of phlebitis, including 51 cases of grade I, 45 cases of grade II, and 177 cases of grade III. Chi-square and Logistic analysis results showed that (Table S3, Table 4) The main factors affecting the occurrence of phlebitis were related to age, insertion site, and type of catheter. Phlebitis was more common in children aged 7-18 years, with under the elbow, or with a 3F. The insertion site under the elbow was the most important risk factor for phlebitis (OR 3.147, 95\% Cl: 2.291-4.323).

4) Analysis of risk factors for catheter broken

5) There are 164 cases occurred catheter broken, of which eight damage in the body, 156 damage out of the body, chi-square test results as shown in Table S4, the main factors influencing the catheter broken include $3 \mathrm{~F}$ catheters $(8.7 \%)$, insertion site under the elbow position (8.3\%), 1-3 years $(7.7 \%)$ and autumn (7.1\%). Logistic results (Table 5) also indicated that the insertion site under the elbow position, autumn, spring, $3 \mathrm{~F}$ catheters were risk factors for catheter damage. The insertion site under the elbow position was the most important risk factor for catheter damage(OR 3.304, 95\% Cl: 2.237-4.878).

6) Analysis of other postoperative complications:

We preliminarily analyzed the characteristics of 32 cases of accidental catheter detachment, 23 was male and 9 was female, including 27 cases of self-removal, 1 case of dressing change, 4 cases of self-removal due to rash and itching. 2 cases of internal rupture of the catheter, one of which occurred after the catheter was blocked, and the other were found at the final extubation. All the 9 cases of in vitro rupture of catheters were disconnected near the Luhe joint, among which 6 cases were male, 3 cases were female, 8 cases were $3 \mathrm{~F}$ catheter, 1 case was $4 \mathrm{~F}$ catheter, and no rupture was found in the Power PICC SOLO catheter. 


\section{Discussion}

We summarized 2974 cases of children with catheterization in our center and analyzed PICC catheterization technology and complications after catheterization. According to the development of technology, PICC insertion technology experienced Non-assistive technique (blind insertion), insertion uses ultrasound, plug technology, plug + ultrasound-guided insertion technology, and we compared the success rate of four technicals, results show that the plug $+B$ ultrasonic technology is the best method of PICC insertion.

PICC-related complications include non-infectious and infectious categories. Infectious complications include infectious phlebitis, insertion site infection, catheter bacterial colonization, and catheter-related bloodstream infection [3]. In contrast, non-infectious complications mainly include mechanical phlebitis, accidental catheter detachment, ectopic location, blockage, damage, drainage, thrombosis, bleeding, nerve, or tendon injury $[4,5]$. The overall incidence of complications associated with PICC catheters was $25-50 \%$ or $4.6-11 / 1000$ catheter days $[6,7]$.

Here, we mainly analyzed the occurrence and risk factors of non-infectious complications in our center. The incidence of non-infectious complications was $0.3-15.2 \%$, which was lower than that reported in the literature [8]. The common postoperative complications in our center included rash (453 cases, 15.2\%), catheter blockage (433 cases, 14.6\%), mechanical phlebitis (282 cases, 9.5\%), and catheter broke (164 cases, $5.5 \%)$. Rare complications included total catheter detachment (34 cases, $1.1 \%$ ), in vivo catheter rupture ( 2 cases, $0.06 \%$ ) and in vitro rupture ( 9 cases, $0.3 \%$ ).

Skin rash is the most common postoperative complication in our center. The results show that the incidence of skin rash is highest in spring, male children, children aged 1-3 years, and children carried Power PICC SOLO catheters. The analysis reason is that spring is the season prone to allergic diseases; male children aged $1-3$ years have high activity and sweat easily under the membrane. The results showed that the incidence of Power PICC SOLO rash was more common than other catheters, which was related to polyurethane material. These results suggest that local care should be strengthened to effectively reduce the occurrence of rashes for young male children who carried Power PICC SOLO catheters.

Catheter blockage is also one of the common complications. A total of 433 cases occurred in our center, with an incidence rate of $14.6 \%$. Combined with the chi-square test and logistic regression analysis, it was found that Power PICC SOLO catheters had the highest rate of catheter blockage and were statistically significant. This is related to the fact that there is no three-way valve in front of the Power PICC SOLO catheter, and blood flow is easy to flow back into the catheter, which is also consistent with literature reports [8]. Therefore, for patients who carried Power PICC SOLO catheters, appropriately increasing the number of flushing is the main method to reduce catheter blockage.

There were 283 cases of phlebitis (14.6\%), which was higher than that reported in the literature [8]. Our results showed that the main factors affecting the occurrence of phlebitis were related to age, insertion 
site, and type of catheter. Children aged 7-18 years old with under the elbow insertion site are more prone to phlebitis. Consider that the diameter of the $4 \mathrm{~F}$ catheter selected for children aged 7-18 years is larger, and under the elbow, insertion site increases the frequency of catheter wear on the vein, it is more likely to have phlebitis. Therefore, avoid placing catheters in areas of flexion is very important to reduce phlebitis [9].

There were 164 cases of catheter broken, including 8 cases of in vivo broken and 156 cases of extracorporeal broken. The analysis results showed that: 1-6 years old, puncture under the elbow, 3F catheter was the main cause of catheter damage. This is related to a large amount of activity in children 1-6 years old, the puncture under the elbow joint is affected by the activity of the elbow joint, and the silicone material is related to the literature reports [8]. Joint movement, extracorporeal catheters are easy to be broken and damaged, suggesting that you should also avoid puncture at the joint site.

We preliminary analyzed the characteristics of the cases of accidental catheter detachment and rupture, and the results showed that children younger than three years old are prone to catheter prolapse, and power PICC solo catheter is not prone to rupture.

\section{Conclusion}

In summary, 1) B-ultrasound plus Seldinger technology can significantly improve the success rate of PICC insertion. 2) The most common non-infectious complications of PICC catheter implantation in children with blood diseases were rash, followed by catheter blockage, mechanical phlebitis, and catheter broke.

3) Male, 1-3 years old, Power PICC solo catheter and spring were risk factors for the rash occurrence, among which Power PICC solo catheter was the most important risk factor for rash. 4) Power PICC solo catheter was the most important risk factor for catheter blockage, followed by the age of 7-11 years old. 5) The insertion site under the elbow was the most important risk factor for phlebitis and catheter damage.

\section{Abbreviations}

PICC, peripherally inserted central catheter; ORs, odds ratios; Cis, confidence intervals; ALL, Acute lymphoblastic leukemia; AML, Acute myeloblastic leukemia; APL, Acute promyelocytic leukemia; HAL, Acute heterozygotic leukemia; MDS: Myelodysplastic syndrome; HPS, Haemophilic cell syndrome; JMML, Juvenile chronic granular mononuclear leukemia; SAA, Sever aplastic anemia; HA, Hemolytic anemia; IST, Immunosuppressive therapy.

\section{Declarations}

\section{Acknowledgements}

None. 


\section{Authors' contributions}

XFZ and HMZ contributed to the conception and design of the study; LXC, YWC, MCW, and SYZ contributed to the acquisition of data. LXC, MW, YT, LT, JXW, and MMY performed the statistical analysis; LXC and HMZ wrote the manuscript; All authors approved the final manuscript for submission.

\section{Funding}

The work of patient information inclusion and statistical analysis was supported by the special construction of vascular access for patients with blood diseases based on international cooperation (201920101402).

\section{Availability of data and materials}

The datasets generated and analyzed during the current study are available from the corresponding author on reasonable request.

\section{Ethics approval and consent to participate}

Our study has been were reviewed and approved by the Clinical Research Ethics Committee of Blood Diseases Hospital \& Institute of Hematology, Chinese Academy of Medical Sciences [HG2020007-EC-1]. Due to the low risk of retrospective project, consent was not required.

\section{Consent for publication}

Not applicable.

\section{Competing interests}

The authors declare that they have no competing interests

\section{References}

1. Huasun. Z. Nursing Science of Intravenous Infusion Therapy. Beijing: People's Military Medical Press. 2007.

2. Society IN. Infusion Nursing Standards of Practice. J Infus Nurs. 2006; 29(1 Suppl):S1-92.

3. Walshe LJ, Malak SF, Eagan J, Sepkowitz KA. Complication rates among cancer patients with peripherally inserted central catheters. J Clin Oncol. 2002; 20(15):3276-81.

4. Cheong K, Perry D, Karapetis C, Koczwara B. High rate of complications associated with peripherally inserted central venous catheters in patients with solid tumours. Intern Med J. 2004; 34(5):234-8.

5. Yap YS, Karapetis C, Lerose S, lyer S, Koczwara B. Reducing the risk of peripherally inserted central catheter line complications in the oncology setting. Eur J Cancer Care (Engl). 2006; 15(4):342-7. 
6. Cardella JF, Cardella K, Bacci N, Fox PS, Post JH. Cumulative experience with 1,273 peripherally inserted central catheters at a single institution. J Vasc Interv Radiol. 1996; 7(1):5-13.

7. Abedin S, Kapoor G. Peripherally inserted central venous catheters are a good option for prolonged venous access in children with cancer. Pediatr Blood Cancer. 2008; 51(2):251-5.

8. Seckold T, Walker S, Dwyer T. A comparison of silicone and polyurethane PICC lines and postinsertion complication rates: a systematic review. J Vasc Access. 2015; 16(3):167-77.

9. society. In: Policies and Procedures for Infusion Nursing. ; 4th edition.

\section{Tables}

Table 1. Comparison of the success rate of different surgical methods

\begin{tabular}{|llllll|}
\hline Group & Title number & Success & Failure & $X^{2}$ & P value \\
\hline A & 550 & $531(96.5 \%)^{*}$ & 19 & 33.613 & 0.00 \\
\hline B & 48 & $47(97.9 \%)$ & 1 & - & - \\
\hline C & 1067 & $1056(98.9 \%)^{*}$ & 11 & - & - \\
\hline D & 1309 & $1305(99.7 \%)^{*}$ & 4 & - & - \\
\hline Total & 2974 & 2939 & 35 & - & - \\
\hline
\end{tabular}

*: Statistically significant difference

Table 2. A binary Logistic regression analysis of the occurrence of rash 


\begin{tabular}{|llll|}
\hline Factor & & OR $(95 \% \mathrm{Cl})$ & P value \\
\hline gender & male & 1.00 & - \\
\hline Age(year) & female & $0.646(0.518-0.804)$ & 0.000 \\
\hline & $4-3$ & 1.00 & - \\
\hline & $7-6$ & $0.705(0.527-0.941)$ & 0.018 \\
\hline Insertion site & above the elbow & 1.00 & 0.000 \\
\hline \multirow{2}{*}{ Catheter type } & under the elbow & - & 0.000 \\
\hline & 4F & $11-18(0.317-0.663)$ & $>0.05$ \\
\hline & Power PICC solo & $3.235(2.255-4.642)$ & 0.000 \\
\hline Season & Spring & 1.00 & - \\
\hline & Summer & $0.877(0.664-1.66)$ & 0.359 \\
\hline & Autumn & $0.722(0.54-0.965)$ & 0.028 \\
\hline & Winter & $0.694(0.515-0.935)$ & 0.016 \\
\hline
\end{tabular}

Table 3. Results of binary logistic regression analysis of catheter occlusion 


\begin{tabular}{|llll|}
\hline Factor & & OR $(95 \% \mathrm{Cl})$ & P value \\
\hline gender & male & 1.00 & - \\
\hline Age(year) & female & - & $>0.05$ \\
\hline & $1-3$ & 1.00 & - \\
\hline & $4-6$ & - & $>0.05$ \\
\hline Insertion site & above the elbow & 1.00 & $>0.05$ \\
\hline \multirow{2}{*}{ Catheter type } & 3F & - & $>0.05$ \\
\hline & under the elbow & - & $>0.05$ \\
\hline & 4F & 1.00 & - \\
\hline Season & Power PICC SOLO & $4.982(3.817-6.504)$ & 0.000 \\
\hline & Spring & 1.00 & - \\
\hline & Summer & - & $>0.05$ \\
\hline & Autumn & - & $>0.05$ \\
\hline & Winter & - & $>0.05$ \\
\hline
\end{tabular}

Table 4. Results of binary logistic regression analysis of phlebitis 


\begin{tabular}{|c|c|c|c|}
\hline Factor & & OR (95\%Cl) & $P$ value \\
\hline \multirow[t]{2}{*}{ Gender } & male & 1.00 & - \\
\hline & female & - & $>0.05$ \\
\hline \multirow[t]{4}{*}{ Age(year) } & $1-3$ & 1.00 & - \\
\hline & $4-6$ & $1.585(1.053-2.384)$ & 0.027 \\
\hline & $7-10$ & $2.101(1.341-3.292)$ & 0.001 \\
\hline & $11-18$ & $2.446(1.508-3.965)$ & 0.000 \\
\hline \multirow[t]{2}{*}{ Insertion site } & above the elbow & 1.00 & - \\
\hline & under the elbow & $3.147(2.291-4.323)$ & 0.000 \\
\hline \multirow[t]{3}{*}{ Catheter type } & $3 F$ & 1.00 & - \\
\hline & $4 \mathrm{~F}$ & $1.242(0.860-1.793)$ & 0.248 \\
\hline & Power PICC SOLO & $0.468(0.220-0.993)$ & 0.048 \\
\hline \multirow[t]{4}{*}{ Season } & Spring & 1.00 & - \\
\hline & Summer & - & $>0.05$ \\
\hline & Autumn & - & $>0.05$ \\
\hline & Winter & - & $>0.05$ \\
\hline
\end{tabular}

Table 5. Results of binary logistic regression analysis of catheter broken 


\begin{tabular}{|llll|}
\hline Factor & & OR $(95 \% \mathrm{Cl})$ & P value \\
\hline Gender & male & 1.00 & - \\
\hline Age(year) & female & - & $>0.05$ \\
\hline & $4-3$ & 1.00 & - \\
\hline & $7-10$ & - & $>0.05$ \\
\hline Insertion site & above the elbow & 1.00 & $>0.05$ \\
\hline \multirow{2}{*}{ Catheter type } & 3F & - & $>0.05$ \\
\hline & under the elbow & $3.304(2.237-4.878)$ & 0.000 \\
\hline Season & Power PICC SOLO & $0.107(0.026-0.450)$ & 0.002 \\
\hline & Spring & 1.00 & - \\
\hline & Summer & $0.504(0.312-0.815)$ & 0.005 \\
\hline & Autumn & $0.933(0.619-1.406)$ & 0.741 \\
\hline & Winter & $0.58(0.363-0.925)$ & 0.022 \\
\hline
\end{tabular}

Figures 


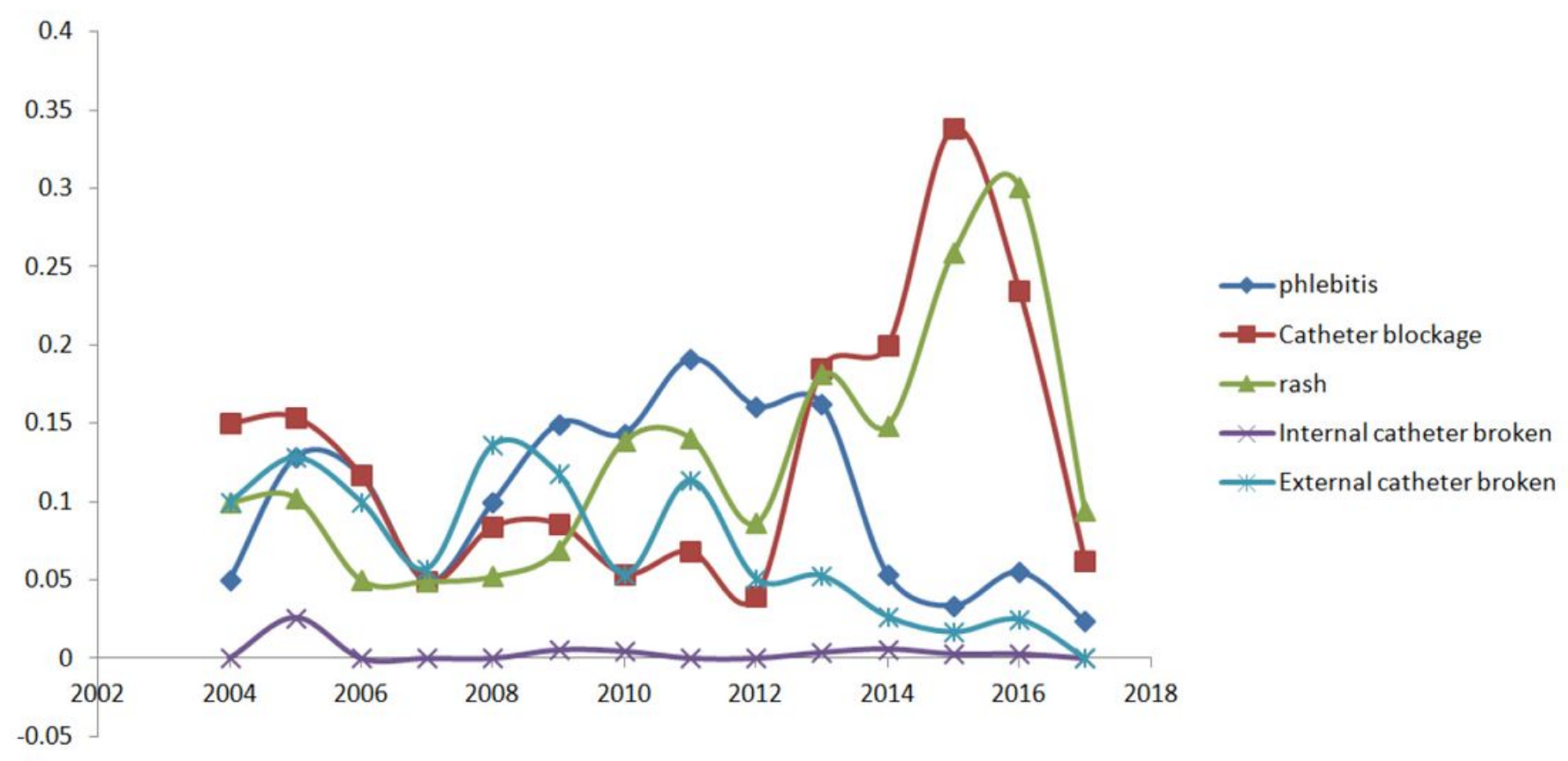

Figure 1

\section{Figure 1}

Complication rate curve from 2004 to 2017

\section{Supplementary Files}

This is a list of supplementary files associated with this preprint. Click to download.

- Supplementarydata.docx

- TableS1S4.docx

- FigureS1.tif 